\title{
ANÁLISE HIDROGEOQUÍMICA COMPARATIVO ENTRE OS AMBIENTES CARBONÁTICOS DO ESTADO DA BAHIA - UMA ABORDAGEM ESTATÍSTICA
}

\author{
COMPARATIVE HYDROGEOCHEMICAL ANALYSIS BETWEEN THE CARBONATE \\ ENVIRONMENTS OF THE STATE OF BAHIA - A STATISTICAL APPROACH
}

\section{Maíra SAMPAIO DA COSTA, Sérgio Augusto de Morais NASCIMENTO, Maria da Conceição RABELO GOMES}

Universidade Federal da Bahia, Instituto de Geociências, Departamento de Geologia. Rua Barão de Jeremoabo s/n, Ondina, Salvador, BA, Brasil. E-mails: mairasampaio42@gmail.com; sergiomn@ufba.br; conceicaorabelo@yahoo.com.br

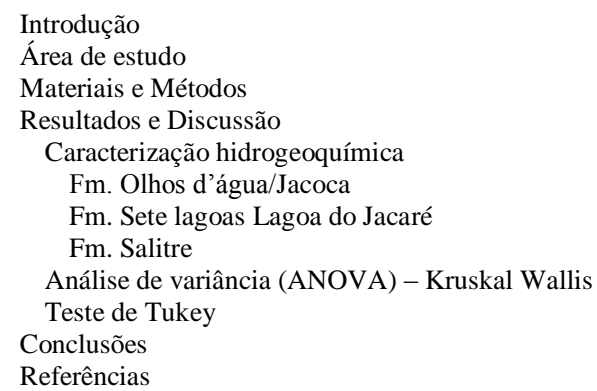

RESUMO - O presente trabalho discute as variações hidrogeoquímicas nas águas subterrâneas dos ambientes carbonáticos do Estado da Bahia. As formações em estudo são: Formação Salitre; formações Sete Lagoas/Lagoa do Jacaré; e Formação Olhos d'água/Jacoca. Os resultados apontam que as formações Olhos d'água/Jacoca apresentam maior concentrações de constituintes químicos, dureza, salinidade e assinatura geoquímica revelando tendências cloretadas e sódicas. A condutividade elétrica apresentou forte correlacão com $\mathrm{Na}^{+}$e $\mathrm{Cl}^{-}$, componentes pluviométricos. As formações Sete Lagoas/Lagoa do Jacaré apresentaram menor concentração de elementos químicos, dureza, salinidade e assinatura geoquímica revelando tendência bicarbonatada cálcica. A condutividade elétrica apresentou forte correlação com o $\mathrm{Na}^{+}, \mathrm{Cl}^{-}, \mathrm{SO}_{4}^{-2}$ e $\mathrm{HCO}_{3}^{-}$, indicando contribuição climática e litológicos. Já a Formação Salitre apresentou assinatura geoquímica revelando tendências bicarbonatada, cálcica e secundariamente magnesiana. A condutividade elétrica apresentou forte correlação com o $\mathrm{Mg}^{2}$, indicando influência de componentes litológicos. Os três ambientes se diferenciaram em relação às médias de $\mathrm{Mg}^{+2} \mathrm{e} \mathrm{HCO}_{3}^{-2}$. Já as formações Sete Lagoas/Lagoa do Jacaré se diferenciam das demais devido às médias de $\mathrm{Ca}^{+2}$. Estes resultados foram associados às diferentes distribuições mineralógicas nas litofácies. As formações Sete Lagoas/Lagoa do Jacaré também se diferenciam da Formação Salitre devido às médias de $\mathrm{SO}_{4}{ }^{-2}$, possivelmente associado à variação de concentração de sulfetos nas áreas.

Palavras-Chave: Geoquímica; Estatística; Aquíferos cársticos; Bahia.

\begin{abstract}
This study discusses the hydrogeochemistry among the carbonate environments of the State of Bahia. The study areas are: Salitre Formation; Sete Lagoas/Lagoa do Jacaré Formations and Olhos d'água/Jacoca Formation. The results suggest that Olhos D'água/Jacoca Formations show a high concentration of physical-chemical elements, hardness, salinity and geochemical signature shows chloride and sodium tendency. The electrical conductivity shows significant correlation with $\mathrm{Na}^{+}$e $\mathrm{Cl}^{-}$, elements of climatic origin. The Lagoas de Sete Lagoas/Lagoa do Jacaré Formations registered the lower concentration of parameters, hardness, salinity and geochemical signature shows bicarbonate and calcium tendency. The electrical conductivity shows significant correlation with $\mathrm{Na}^{+}, \mathrm{Cl}^{-}, \mathrm{SO}^{-2}{\mathrm{e} \mathrm{HCO}_{3}}^{-}$, suggesting a shared contribution between the elements of climatic and lithological origin. In the Salitre Formation the geochemical signature shows bicarbonate, calcium and magnesium tendency. The electrical conductivity shows significant correlation with $\mathrm{Mg}+^{2}$, suggesting influence of elements of lithological. The three environments differ themselves according to the mean variations of $\mathrm{Mg}^{+2}$ and $\mathrm{HCO}_{3}$. Sete Lagoas/Lagoa do Jacaré Formations differ themselves of the others according to mean variation of $\mathrm{Ca}^{+2}$. These results are related with different mineral distributions in lithofacies. Sete Lagoas/Lagoa do Jacaré Formation also differ of Salitre Formation according to mean variation of $\mathrm{SO}_{4}^{-2}$, possibly associated with concentration variation of sulfide in the area.
\end{abstract}

Key words: Geochemistry; Statistics; Carbonate aquifer; Bahia.

\section{INTRODUÇÃO}

A hidrogeoquímica é um ramo da ciência que trata da composição química das águas, de suas variações e das causas dessas variações, estabelecendo uma relação com o meio físico (Gonçalves, 2002). Segundo Mestrinho (2008), um pré-requisito nos estudos hidrogeoquímicos é o entendimento dos processos que ocorrem associados às águas naturais e os problemas de contaminação, pois águas poluídas promovem mudanças no estado físico e químicos das soluções.

A composição das águas subterrâneas nos 
sistemas cársticos está vinculada principalmente aos processos químicos motivados pela interação água-rocha. Os minerais constituintes de rocha apresentam suas próprias propriedades de solubilidade, afetam na saturação e diversidade de constituintes em solução. Os íons que são incorporados ao meio influenciam em suas propriedades, como salinidade, cor, sabor e dureza e definem a classificação e afetam na qualidade das águas (Santos, 2013; Mestrinho, 2008). Já a mobilidade química dos componentes dissolvidos em solução, está relacionada a fatores como: capacidade de troca iônica, processos de ligações com a litosfera e biosfera e a própria solubilidade, está dependendo do potencial iônico de cada íon (Ramage, 2005).

As rochas calcárias são mais propicias aos processos de dissolução do que a maior parte das litologias, produzindo paisagens cársticas pronunciadas e gerando grande interesse cientifico (Silva, 2008). Do ponto de vista socioeconômico, os processos de desenvolvimento social colaboraram para uma elevação do consumo de água subterrânea, que tem como uma de suas fontes os ambientes cársticos, fazendo surgir a necessidade de um controle sistemático de sua qualidade (Nossa, 2011). Os sistemas cársticos aqui estudados estão localizados no Estado da Bahia e situados geologicamente no Supergrupo São Francisco, que data do Proterozoico Superior. Este estudo tem como objetivo estabelecer diferenciações hidrogeoquímica entre tais ambientes carbonáticos a partir de ferramentas estatísticas, apresentando relevância cientifica para a gestão dos recursos hídricos subterrâneos.

\section{ÁREA DE ESTUDO}

A área de estudo abrange três ambientes carbonáticos localizados no Estado da Bahia: Formação Salitre (Grupo Una), Formações Sete Lagoa/Lagoa do Jacaré (Grupo Bambuí) e Formações Olhos d'água/Jacoca (Grupo VazaBarris/Miaba) (Negrão, 2007; Oliveira et al., 2007) (Figura 1). O Grupo Una teve sua deposição inicial
(Formação Bebedouro) relacionada a um evento glacial no início do Neoproterozoico. Após, a implantação gradual de um clima semiárido permitiu a dissolução das geleiras, que contribuiu para elevar o nível das marés, gerando as condições para deposição das unidades carbonáticas da Formação Salitre (Sampaio et al., 2001).

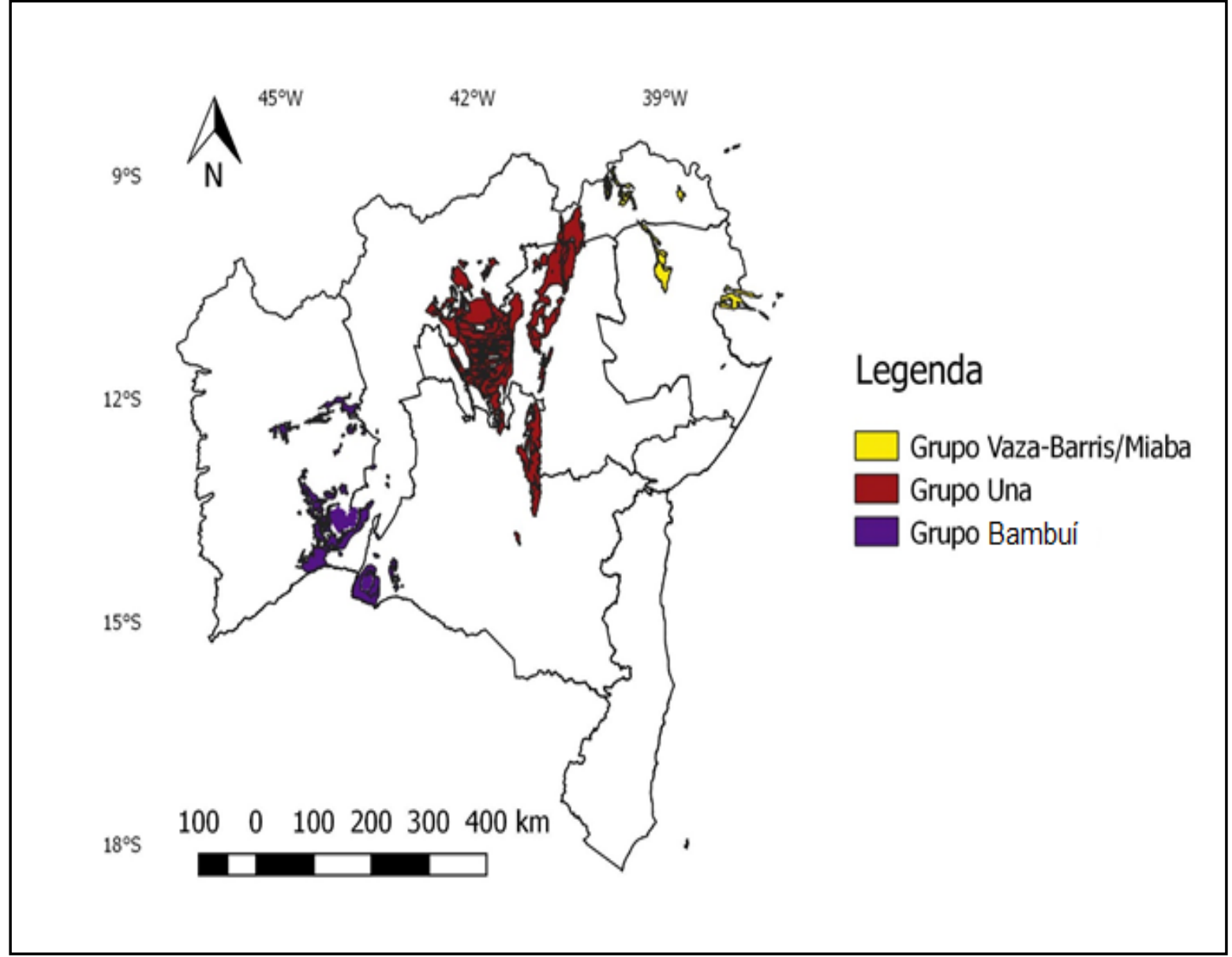

Figura 1 - Mapa de domínios carbonáticos do Estado da Bahia. 
Misi \& Souto (1975) distinguiu seis unidades estratigráficas que, da base para o topo, compõemse da seguinte forma: Unidade $C$ constituída por dolomitos e calcários dolomíticos vermelhos e argilosos; Unidade B por calcários cinza-claro laminados e estratificados; Unidade B1 por dolarenitos com teepees (fendas de ressecamento); Unidade A por argilitos calcíferos, margas e siltitos; e Unidade A1 por calcários oolíticos e cacilutitos pretos (Figura 2)



Figura 2 - Coluna estratigráfica proposta para a Formação Salitre Adaptado de Misi \& Silva (1996).

A deposição dos sedimentos carbonáticos do Grupo Bambuí está relacionada ao mesmo evento de deglaciação que originou as unidades do Grupo Una e este ambiente está dividido em Fm. Sete Lagoas na base, e Fm. Lagoa do Jacaré mais ao topo. A Formação Sete Lagoas é constituída por calcários laminados, calcários, dolomitos estromatolíticos, doloarenitos e brechas dolomíticas, depositados em plataforma carbonática (Nobre-Lopes, 1995; Lima, 1997). Já a Formação Lagoa do Jacaré é caracterizada pela associação de depósitos carbonáticos de retrabalhamento, principalmente calcarenitos oolíticos a pisolíticos, calciruditos e doloruditos, intercalados com siltitos e raras bioconstruções carbonáticas (Lima, 2005) (Figura 3).

\begin{tabular}{|c|c|c|}
\hline $\begin{array}{c}\text { Coluna } \\
\text { Litoestratigráfica }\end{array}$ & Formação & Associação litológica \\
\hline \multirow{4}{*}{ 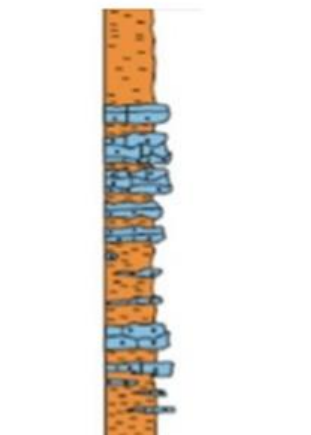 } & $\begin{array}{l}\text { Serra da } \\
\text { Saudade }\end{array}$ & $\begin{array}{l}\text { Pelitos, siltitos, folhelhos. } \\
\text { Cálcario subordinado }\end{array}$ \\
\hline & $\begin{array}{l}\text { Lagoa do } \\
\text { Jacaré } 2\end{array}$ & $\begin{array}{l}\text { Calcarenito com pelito } \\
\text { subordinado }\end{array}$ \\
\hline & $\begin{array}{l}\text { Lagoa do } \\
\text { Jacaré } 1\end{array}$ & $\begin{array}{l}\text { Pelito, marga e } \\
\text { calcarenito }\end{array}$ \\
\hline & $\begin{array}{l}\text { Serra da } \\
\text { Santa Helena }\end{array}$ & $\begin{array}{l}\text { Pelito, siltito e } \\
\text { calcarenito subordinado }\end{array}$ \\
\hline \multirow[t]{5}{*}{$\frac{12}{823}$} & Sete Lagoas 3 & $\begin{array}{l}\text { Calcário dolomítico, } \\
\text { dolarenito e dolomito }\end{array}$ \\
\hline & Sete Lagoas 2 & $\begin{array}{l}\text { Calcirudito (teepee), } \\
\text { calcarenito e calcário }\end{array}$ \\
\hline & Sete Lagoas 1 & Dolomitos, margas e pelitos \\
\hline & Jequitai & Siltito, argilito e diamictito \\
\hline & \multicolumn{2}{|l|}{$\begin{array}{l}\text { Pré-cambriano } \\
\text { Arqueano }\end{array}$} \\
\hline
\end{tabular}

Figura 3 - Coluna estratigráfica do Grupo Bambuí. Adaptado de Misi et al. (2007). 
O Grupo Vaza-Barris/Miaba está inserido na Faixa Sergipana no limite nordeste do Cráton do São Francisco e foi formado durante o ciclo Brasiliano/Pan-Africano (Barbosa \& Dominguez, 1996). As espessas camadas carbonáticas se acumularam em bacias marinhas intracratônicas (epicontinentais) e são divididas em Fm. Jacoca mais a base, associado ao Grupo Miaba, e Fm. Olhos d'Água mais ao topo, associado ao Grupo
Vaza-Barris (Figura 4) (Gonçalves, 2014).

A Fm Jacoca é composta, da base para o topo, por metadolomitos, sucedidos por metacalcários, metadolomitos com intercalações de sílex (Barbosa \& Dominguez, 1996). Já a Formação Olhos d'Água é composta de metacalcários (calcarenitos) com intercalações de filitos calcíferos, metadolomitos e brechas intraformacionais (Uhlein et al., 2011).

\begin{tabular}{|c|c|c|c|}
\hline \multicolumn{2}{|c|}{$\begin{array}{c}\text { Coluna } \\
\text { Litoestratigráfica }\end{array}$} & Unidades & Associação litológica \\
\hline \multirow{4}{*}{$\begin{array}{l}\text { Grupo } \\
\text { Vaza } \\
\text { Barris }\end{array}$} & \multirow{2}{*}{ 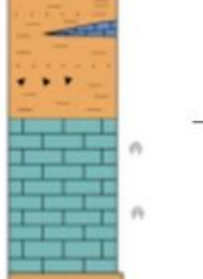 } & $\begin{array}{l}\text { Fm. Frei Paulo- } \\
\text { Ribeirópolis }\end{array}$ & $\begin{array}{l}\text { Metassiltito, metapelito, quartzito, } \\
\text { metadiamictito e metacarbonato }\end{array}$ \\
\hline & & Fm. Olhos d' água & $\begin{array}{l}\text { Metacalcário (calcarenitos) com intercalações } \\
\text { de filitos calcíferos, sílex, metadolomito } \\
\text { e brechas intraformacionais }\end{array}$ \\
\hline & & $\begin{array}{l}\text { Fm. Capitão } \\
\text { Palestina }\end{array}$ & $\begin{array}{l}\text { Metadiamictitos maciços com matriz argilo-siltosa } \\
\text { e arenosa, intercalações de metagrauvaca, } \\
\text { metaritmito, filito e quartzito. }\end{array}$ \\
\hline & & Fm. Jacoca & $\begin{array}{l}\text { Metacalcário/ Metadolomito com intercalações } \\
\text { de sílex }\end{array}$ \\
\hline \multirow{2}{*}{$\begin{array}{l}\text { Grupo } \\
\text { Miaba }\end{array}$} & 要员里 & Fm. Jacarecica & $\begin{array}{l}\text { Metadiamictito maciço e camadas alternadas } \\
\text { de metagrauvaca e metapelito }\end{array}$ \\
\hline & $1++$ & Fm. Itabaiana & $\begin{array}{l}\text { Quartzitos finos a grossos, conglomeráticos, } \\
\text { arcoseanos, metassiltito }\end{array}$ \\
\hline
\end{tabular}

Figura 4 - Coluna estratigráfica dos Grupos Miaba e Vaza-Barris, Faixa Sergipana, com descrição de litofácies e respectivos sistemas deposicionais. Adaptado de Uhlein et al. (2011) apud Humphrey \& Allard (1969), Silva Filho et al. (1979), Sial et al. (2010) e Uhlein et al. (2011).

\section{MATERIAIS E MÉTODOS}

Neste trabalho foram utilizadas informações hidroquímicas de 364 poços tubulares, sendo 12 da Fm Olhos d'água/Jacoca, 122 da Fm Sete Lagoas/Lagoa do Jacaré e 230 da Fm Salitre.

Os dados foram obtidos a partir do sistema de informações de água subterrânea da Companhia de Pesquisa e Recursos Minerais (CPRM). As análises químicas seguiram nos manuais Standard Methods for the Examination of Water and Wastewater (APHA, 1995) e na espectrometria de emissão ótica (ICP-OES).

Devido a distribuição assimétrica dos dados analíticos, foram empregadas técnicas estatísticas não paramétricas.

Os cálculos estatísticos realizados utilizaram os programas do Excel, Action e Diagrammes. No Excel (2013) confeccionou-se tabelas e no Action (Statsoft 3.0) processou-se resultados estatísticos básicos e avançados: teste de Tukey, regressão múltipla e análise de variância (ANOVA) pelo método de Kruskal Wallis. Já no Diagrammes foram obtidos os Diagramas de Schoeller-Berkaloff que apontam as tendências geoquímicas das águas.

A análise de variância (ANOVA), ferramenta estatística que testa a igualdade de médias populacionais, foi utilizada com a finalidade de diferenciar os ambientes carbonáticos em estudo. O teste de Tukey, método complementar da ANOVA, foi empregado para identificar os parâmetros dispares (Field, 2009).

Já a regressão múltipla foi utilizada com a finalidade de avaliar a associação entre a condutividade elétrica (CE) e os parâmetros estudados (Bauer, 2007).

A escolha desses métodos estatísticos se 
fundamentou na frequente empregabilidade dos mesmos em pesquisas de qualidade das águas e hidrogeoquímica (Cooper et al., 2018; Nascimento et al., 2016; Carrera-Villacrés et al., 2016). A ANOVA e o teste de Tukey foram aplicados utilizando parâmetros químicos maiores, que melhor caracterizam às rochas carbonáticas, sendo eles, $\mathrm{Mg}^{+2}, \mathrm{Ca}^{+2}, \mathrm{SO}_{4}{ }^{-2} \mathrm{e}$ $\mathrm{HCO}_{3}{ }^{-}$. Na realização das demais técnicas foram empregados os parâmetros: $\mathrm{Na}^{+}, \mathrm{K}^{+}$, $\mathrm{Mg}^{+2}, \quad \mathrm{Ca}^{+2}, \quad \mathrm{HCO}_{3}^{-}, \quad \mathrm{SO}_{4}^{-2}, \mathrm{NO}_{3}^{-}, \mathrm{Cl}$, condutividade elétrica (CE) e $\mathrm{pH}$.

\section{RESULTADOS E DISCUSSÕES}

\section{Caracterização Hidrogeoquímica}

Os valores de $\mathrm{pH}$ nos três ambientes em estudo apresentam tendências alcalinas com mediana nas Fm. Olhos d'água/Jacoca e Fm. Salitre de 7,4 e nas Fm. Sete Lagoas/Lagoa do Jacaré de 7,5.

Para esses intervalos de variação, Apello \& Postma (2005) afirmam que o $\mathrm{pH}$ possui controle sobre a dissolução de $\mathrm{HCO}_{3}^{-}$, favorecendo a presença desse elemento em solução (mediana de $\mathrm{HCO}_{3}{ }^{-}$na $\mathrm{Fm}$. Olhos d'água/Jacoca $=425,5 \mathrm{mg} / \mathrm{l} ; \mathrm{Fm}$. Sete Lagoas/Lagoa do Jacaré $=269,5 \mathrm{mg} / \mathrm{l}$; e Fm. Salitre $=288 \mathrm{mg} / \mathrm{l})$.

Resultados semelhantes foram encontrados por Guerra (1986), Gonçalves (2014), Galvão et al. (2017) e Gonçalves (2018) para a Fm. Sete Lagoas/Lagoa do Jacaré; e por Nossa (2011), Sales (2017), Santos (2017) e Cunha (2018) para a Fm Salitre.

Custodio e Llamas (2011) estabelece a classificação de dureza em: valores abaixo de $50 \mathrm{mg} / \mathrm{l} \mathrm{de} \mathrm{CaCO}_{3}$ (leve), entre $50-100 \mathrm{mg} / 1$ (moderadamente dura), entre 100-200 mg/l (dura) e acima de $200 \mathrm{mg} / \mathrm{l}$ (muito dura). Os três ambientes em estudo apresentam águas com elevada dureza. A tabela 1 indica as medianas concentrações e os intervalos de variações dos parâmetros físicos e químicos em estudo.

Tabela 1 - Concentração em mg/l dos parâmetros em estudo nos ambientes carbonáticos.

\begin{tabular}{c|c|c|c|c|c|c}
\hline & \multicolumn{5}{|c}{ Intervalo da mediana e variação das concentrações iônicas (mg/l) } \\
\hline Parâmetros & Fm. Sete Lagoas / Lagoa do Jacaré & \multicolumn{2}{|c}{ Fm. Salitre } & \multicolumn{2}{c}{ Fm Olhos d'água/Jacoca } \\
\hline $\mathbf{C a}^{+\mathbf{2}}$ & 213,5 & $133-292,3$ & 424,2 & $234,5-515,5$ & 379,25 & $288-716,25$ \\
\hline $\mathbf{N a}^{+}$ & 33,3 & $11,6-81,8$ & 78,81 & $21,28-93,4$ & 337,5 & $241-762,5$ \\
\hline $\mathbf{M g}^{+\mathbf{2}}$ & 14,9 & $7-22,8$ & 71,66 & $39,7-97,3$ & 111 & $66,1-141,5$ \\
\hline $\mathbf{K}^{+}$ & 2 & $1,47-3$ & 4,11 & $1,8-5,5$ & 2 & $1,47-3$ \\
\hline Dureza & 264 & $193,25-405,3$ & 722,19 & $424,25-937$ & 965 & $558,3-1199$ \\
\hline $\mathbf{S O 4}^{-2}$ & 28,5 & $5,1-81,9$ & 204,48 & $39,92-187,8$ & 163,5 & $85,5-276,3$ \\
\hline $\mathbf{N O 3}^{-2}$ & 0,4 & $0,102-1,3$ & 10,74 & $0,545-13,4$ & 8,47 & $3,9-15,9$ \\
\hline $\mathbf{C l}^{-}$ & 21,9 & $9,54-58,5$ & 257,6 & $66,02-315,3$ & 601 & $355-1471$ \\
\hline $\mathbf{H C O}^{-}$ & 269,5 & $219,75-333,3$ & 295,77 & $248-249,3$ & 425,5 & $355-461$ \\
\hline $\mathbf{C E}^{-2}$ & 706 & $488,5-998,6$ & 1638,9 & $958,9-2045$ & 3270,5 & $1956,5-5427,5$ \\
\hline
\end{tabular}

Os diagramas de Schoeller-Berkaloff (Figuras 5 a 7) apresentam as distribuições iônicas em cada formação geológica estudada e a figura 8 mostra a mediana das concentrações dos elementos químicos nos ambientes carbonáticos.

\section{Fm. Olhos d'água/Jacoca}

$\mathrm{O}$ valor de $\mathrm{CaCO}_{3}$ é igual a $965 \mathrm{mg} / \mathrm{l}$, sendo o ambiente com maior dureza.

Em geral, apresenta as maiores concentrações componentes químicos e físicos, exibindo mediana de condutividade elétrica (CE) igual a $3270,5 \mu \mathrm{S} / \mathrm{cm}$ que refletem na maior salinidade dessa solução (Santos, 2008) (Figura 8 e Tabela 1).
O diagrama de Schoeller-Berkaloff aponta, na figura 5, a assinatura geoquímica das águas subterrâneas das Fm. Olhos d'água/Jacoca indicando tendências sódica, cloretada e cálcica das águas.

$\mathrm{O} \mathrm{Na}^{+}$(mediana $\left.=337.5 \mathrm{mg} / \mathrm{l}\right)$ e $\mathrm{Cl}^{-}$(mediana $=601 \mathrm{mg} / \mathrm{l})$ apresentando elevadas concentrações na solução justificando sua influência sobre essa, tendo origem associada as águas meteóricas (Santos, 2013). Já o $\mathrm{Ca}^{+2}$ (mediana $=379,2 \mathrm{mg} / \mathrm{l})$, segundo Uhlein et al. (2011), ocorre em função da litologia calcária presente na área contendo mineiras de calcita e dolomita. 


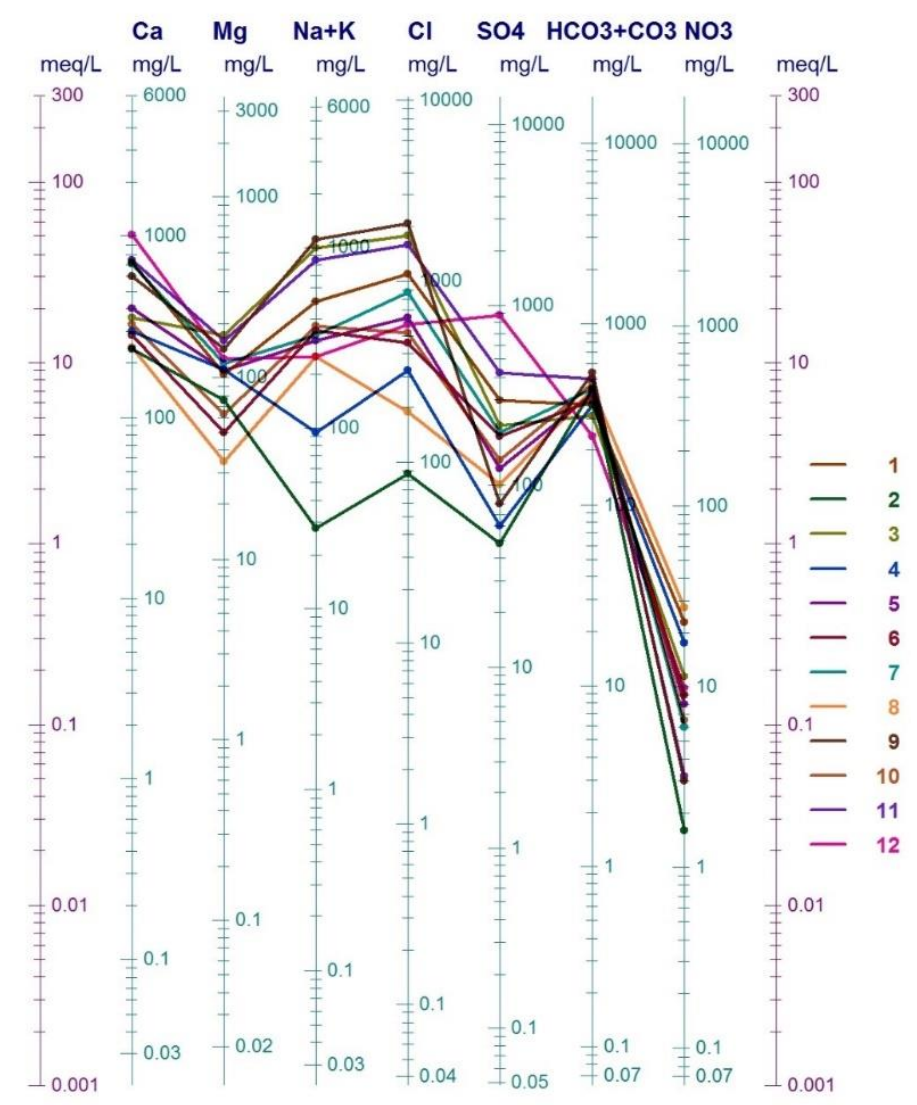

Figura 5 - Diagrama Schoeller-Berkaloff para águas das. Fm. Olhos d'água/Jacoca. Poços: P-1, P-2, P-3, P-4, P-5, P-6, P-7, P-8, P-9, P-10, P-11, P-12.

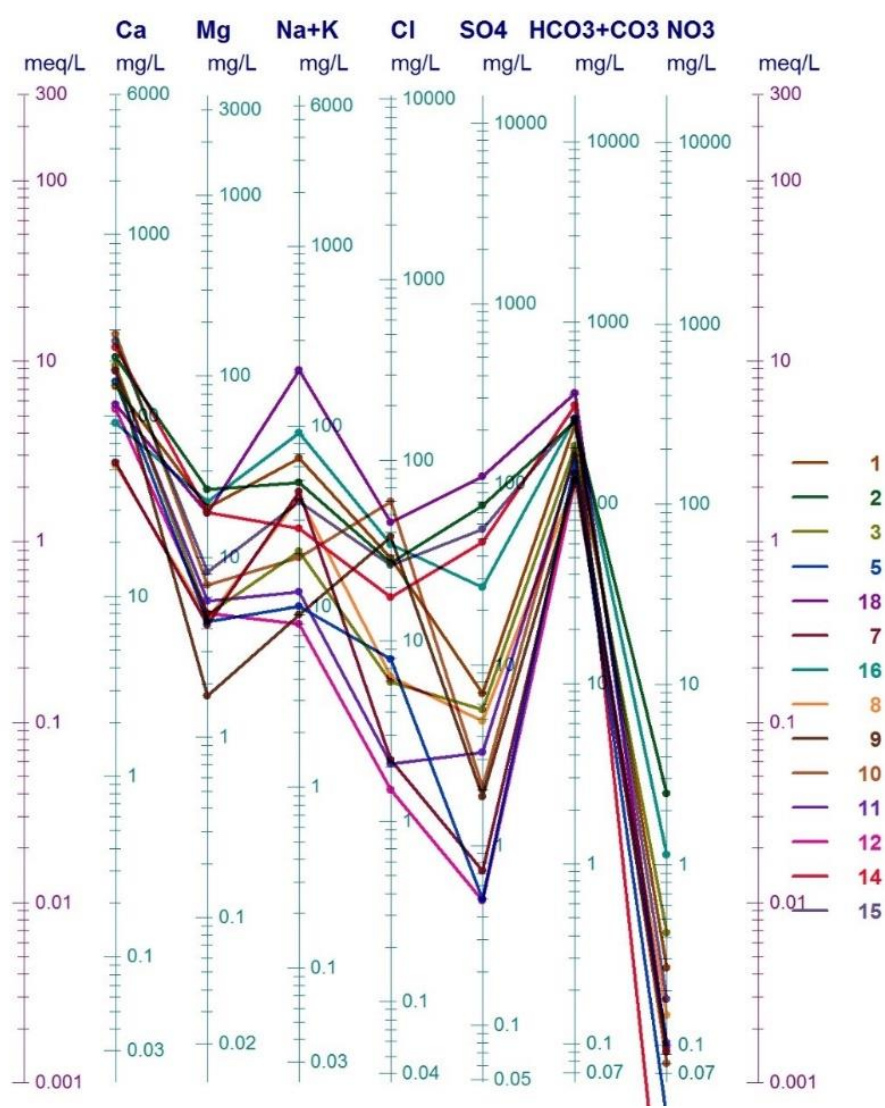

Figura 6 - Diagrama Schoeller-Berkaloff para águas das. Fm. Sete Lagoas/Lagoa do Jacaré. Poços: P-1, P-2, P-3, P-5, P7, P-8, P-9, P-10, P-11, P-12, P-14, P-15, P-16 e P-18. 


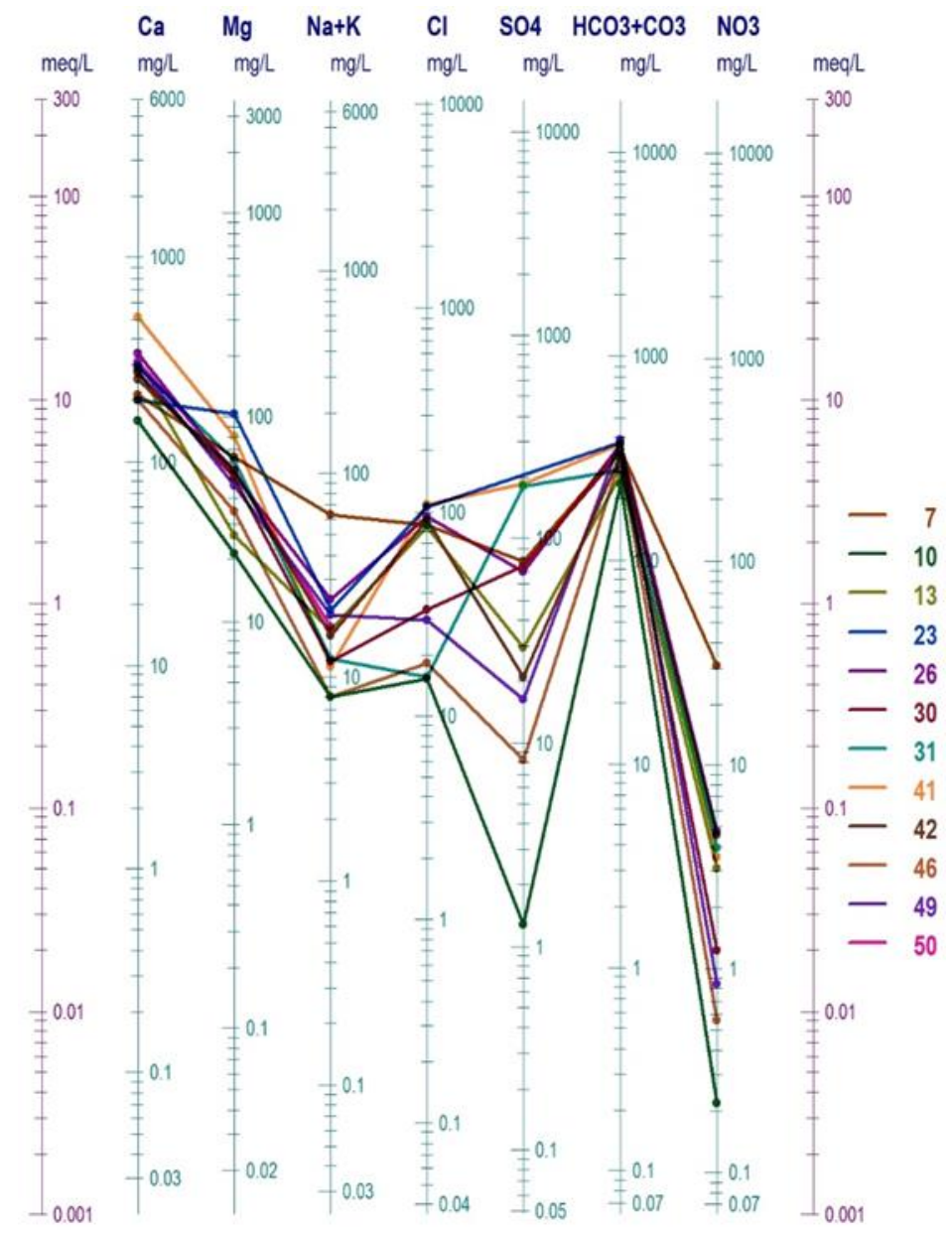

Figura 7 - Diagrama Schoeller-Berkaloff para águas da. Fm. Salitre. Poços: P-7, P-10, P-13, P-23, P-26, P-30, P-31 P41, P-42, P-46, P-49, P-50.



Figura 8 - Mediana de concentração dos elementos químicos nos ambientes carbonáticos. Unidades de medidas: $\mathrm{HCO}_{3}{ }^{-}$ $, \mathrm{Cl}^{-}, \mathrm{Mg}^{+2}, \mathrm{Ca}^{+2}, \mathrm{~K}^{+}, \mathrm{Na}^{+}$e $\mathrm{SO}_{4}{ }^{-2} \mathrm{em} \mathrm{mg} / \mathrm{l}, \mathrm{CE}$ em $\mu \mathrm{S} / \mathrm{cm}$. 


\section{Fm. Sete Lagoas/Lagoa do Jacaré}

$\mathrm{O}$ valor de $\mathrm{CaCO}_{3}$ é igual a $213,5 \mathrm{mg} / \mathrm{l}$, sendo o ambiente com menor dureza. Em geral, apresenta as menores concentrações dos elementos químicos e físicos, assim como menor $\mathrm{CE}($ mediana $=706 \mu \mathrm{S} / \mathrm{cm})$, refletindo na menor salinidade dessa solução (Figura 8 e Tabela 1). Resultados semelhantes foram encontrados por Galvão et al. (2017).

Neste ambiente, o diagrama de SchoellerBerkaloff aponta, na figura 6 , a assinatura geoquímica com tendência principal bicarbonatada e cálcica nas águas subterrâneas, e secundariamente sódica e cloretada. Segundo Gonçalves (2014), Gonçalves (2018) e Carvalho (2018), a influência de $\mathrm{Ca}^{+2}$ (mediana $=213,5$ $\mathrm{mg} / \mathrm{l})$ e $\mathrm{HCO}_{3}{ }^{-}(269,5 \mathrm{mg} / \mathrm{l})$ na solução refletem a presença dos calcário e dolomitos na Fm. Sete Lagoas/Lagoa do Jacaré (Figura 3). A influência dos íons de $\mathrm{Cl}^{-}$(mediana $=21,9 \mathrm{mg} / \mathrm{l}$ ), associamse ao controle das águas meteóricas sobre a solução (Santos, 2008; Carvalho, 2018; Gonçalves, 2018). Já a presença do $\mathrm{Na}^{+}$ (mediana $=33 \mathrm{mg} / \mathrm{l})$, segundo Guerra (1986) e Diniz (2006), relaciona-se ao intemperismo químico de plagioclásio no ambiente.

\section{Fm. Salitre}

$\mathrm{O}$ valor de $\mathrm{CaCO}_{3}$ é igual a $354,1 \mathrm{mg} / \mathrm{l}$, indicando intermediária dureza dentre os ambientes em estudo (Figura 8 e Tabela 1). Em geral, apresenta intermediária concentração dos elementos químicos e físicos, exibindo mediana de CE igual a $1400 \mu \mathrm{S} / \mathrm{cm}$, proporcionando uma intermediária salinidade (Santos, 2008). Guerra (1986) e Nossa (2011) encontram valores semelhantes.

O diagrama de Schoeller-Berkaloff indica, na figura 7, a assinatura geoquímica com tendências bicarbonatadas, cálcicas e secundariamente magnesianas nas águas subterrâneas.

Nossa (2011) afirma que a influência de $\mathrm{Mg}^{+2}$ ocorre em função da dissolução da dolomita $\left(\mathrm{CaMg}\left(\mathrm{CO}_{3}\right)_{2}\right)$, relacionada sobretudo à Unidade Nova América Inferior, (Figura 2) que é composta principalmente por dolomitos. Villaneuva (2014) também constatou influência de $\mathrm{Mg}^{+2}$ na solução desse ambiente. Já os íons de $\mathrm{Ca}^{+2}$ e $\mathrm{HCO}_{3}^{-}$são componentes comuns das rochas carbonáticas da Fm. Salitre (Nossa, 2011; Villaenuva, 2014; Sales, 2017; Cunha, 2018).

\section{Regressão Múltipla}

Este método é utilizado para identificar e explicar a participação dos elementos químicos na variação da condutividade elétrica (CE), responsável pela salinidade da solução (Field, 2009). A condutividade elétrica foi utilizada como variável dependente enquanto os demais componentes utilizados nessa pesquisa foram utilizados como variáveis independentes $\left(\mathrm{Na}^{+}\right.$, $\mathrm{K}^{+}, \quad \mathrm{Mg}^{+2}, \quad \mathrm{Ca}^{+2}, \quad \mathrm{HCO}_{3}^{-}, \quad \mathrm{SO}_{4}^{-2}, \quad \mathrm{Cl}^{-} \mathrm{e}$ condutividade elétrica). $\mathrm{O}$ nível de significância adotado no presente estudo foi $\alpha=0,05$ (5\%).

\section{Fm Olhos d'água / Jacoca}

$\mathrm{O} \mathrm{Ca}^{+2}$ e o $\mathrm{Cl}^{-}$apresentaram valor-p próximo a zero (Tabela 2), indicando rejeição da hipótese $\mathrm{H}_{0}$. A equação linear múltipla obtida nesse caso foi: $\mathrm{CE}=858+1,3 \mathrm{Na}^{+}+2,3 \mathrm{~K}^{+}+1,4 \mathrm{Ca}^{+2}+2,1$ $\mathrm{Cl}^{-}-3,0 \mathrm{Mg}^{+2}$, indicando que o $\mathrm{Na}^{+}, \mathrm{K}^{+}$e $\mathrm{Cl}^{-}$ contribuem em cerca de $80 \%$ para a condutividade elétrica da solução, enquanto que o $\mathrm{Ca}^{2+}$ contribuiu $20 \%$. $\mathrm{O} \mathrm{Na}^{+} \mathrm{e} \mathrm{Cl}^{-}$das águas possuem provável origem na pluviometria da área (Santos, 2013) o que sugere maior influência climática no incremento da salinidade. Tendência indicada pelo Diagrama SchoellerBerkaloff.

\section{Fm Sete Lagoas/Lagoa do Jacaré}

Neste ambiente, o $\mathrm{Na}^{+}, \mathrm{Cl}^{-}, \mathrm{SO}_{4}{ }^{-2}$ e $\mathrm{HCO}_{3}{ }^{-}$ apresentam valor-p próximos a zero, indicando rejeição da hipótese $\mathrm{H}_{0}$. A equação linear múltipla obtida foi: $\mathrm{CE}=91+0,5 \mathrm{Na}+1,6 \mathrm{HCO}_{3}{ }^{-}$ $+2,7 \mathrm{Cl}^{-}+1,4 \mathrm{SO}_{4}^{-2}$ indicando que o $\mathrm{Na}^{+}$e $\mathrm{Cl}^{-}$, contribuíram em $52 \%$ para a salinidade das águas subterrâneas, enquanto que o $\mathrm{SO}_{4}{ }^{-2}$ e $\mathrm{HCO}_{3}{ }^{-}$ contribuíram com $48 \%$. As análises estatísticas de Gonçalves (2014) indicaram resultados semelhantes quanto ao $\mathrm{Na}^{+} \mathrm{e} \mathrm{Cl}^{-}$.

Segundo Gonçalves (2014) a presença de $\mathrm{SO}_{4}$

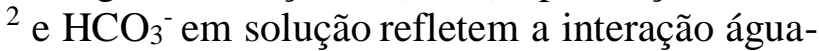
rochas carbonáticas da área. Resultados semelhantes foram encontrados por Guerra (1986) e Kim et al (2011) afirmam que as concentrações de $\mathrm{Cl}^{-}$no aquífero associam-se aos fatores climáticos e a taxa de evaporação local. Já a presença do $\mathrm{Na}^{+}$, segundo Guerra (1986) e Diniz (2006), relaciona-se ao intemperismo químico de plagioclásio no ambiente. Assim, sugere-se contribuição tanto de elementos químicos de origem litológica quanto meteórica para a salinidade da solução.

\section{Fm Salitre}

$\mathrm{O} \mathrm{Na}^{+}, \mathrm{Cl}^{-}, \mathrm{Mg}^{2}$ e $\mathrm{HCO}_{3}^{-}$apresentaram valor- $\mathrm{p}$ próximos a zero, mostrando rejeição da hipótese $\mathrm{H}_{0}$ com relação a condutividade elétrica (CE) (Tabela 2). A equação linear múltipla obtida nesse caso foi: $\mathrm{CE}=780+2 \mathrm{Na}^{+}+9 \mathrm{Mg}^{+2}$ 
$+2 \mathrm{Cl}^{-}$, indicando que o $\mathrm{Na}^{+}$e o $\mathrm{Cl}^{-}$contribuíram em $30 \%$ para a condutividade elétrica, enquanto que o $\mathrm{Mg}+{ }^{2}$ contribuiu cerca de $70 \%$.

Nossa (2011) afirma que as elevadas concentrações de $\mathrm{Mg}^{2}{ }^{2}$ em solução ocorrem devido à dissolução de dolomita $\left(\mathrm{CaMg}(\mathrm{CO} 3)_{2}\right)$, associada principalmente a Unidade Nova América Inferior. Villaneuva (2014) também constatou elevadas concentrações de $\mathrm{Mg}{ }^{2}$ nas águas do aquífero Salitre. Esse resultado indica maior influência de componentes de origem litológica na salinidade da solução.

Tabela 2 - Regressão múltipla dos ambientes carbonáticos em estudo.

\begin{tabular}{c|c|c}
\hline $\begin{array}{c}\text { Fm Sete Lagoas / } \\
\text { Lagoa do Jacaré }\end{array}$ & Coeficientes & valor-P \\
\hline Interseção & 90,7 & $1,6.10^{-5}$ \\
\hline Sódio & 0,5 & $1,9.10^{-4}$ \\
\hline Bicarbonato & 1,6 & $2.10^{-8}$ \\
\hline Magnésio & $2.10^{-2}$ & 0,9 \\
\hline Cloreto & 2,7 & $6,5.10^{-9}$ \\
\hline Sulfato & 1,4 & $8.10^{-11}$ \\
\hline Fm Salitre & Coeficientes & valor-P \\
\hline Interseção & 779,7 & $1,6.10^{-15}$ \\
\hline Sódio & 2,0 & $1,4.10^{-15}$ \\
\hline Bicarbonato & $-1,3$ & $1,3.10^{-5}$ \\
\hline Magnésio & 9,4 & $6,5.10^{-10}$ \\
\hline Cloreto & 1,6 & $0,9.10^{-8}$ \\
\hline Fm Olhos d'água / & Coeficientes & valor-P \\
\hline Jacoca & & \\
\hline Interseção & 857,9 & $3.10^{-3}$ \\
\hline Sódio & 1,3 & $1,5.10^{-1}$ \\
\hline Potássio & 2,3 & 0,3 \\
\hline Cálcio & 1,5 & $5,7.10^{-4}$ \\
\hline Magnésio & $-3,0$ & 0,3 \\
\hline Cloreto & 2,1 & $4,5.10^{-3}$ \\
\hline
\end{tabular}

\section{Análise De Variância (Anova) - Kruskal- Wallis}

Para os cálculos de análise de variância, foram utilizados os componentes $\mathrm{Ca}^{+2}, \mathrm{Mg}^{+2}, \mathrm{HCO}^{-} \mathrm{e}$ $\mathrm{SO}_{4}{ }^{-}$e partir da hipótese nula (Ho) adotada, que admite que quando as médias populacionais são iguais entre os ambientes $\left(\mathrm{Sx}^{2}\right)$ e dentro deles $\left(\mathrm{Sp}^{2}\right)$, as populações estudadas apresentam semelhanças significativas (Field, 2009).

A razão $\mathrm{F}$ obtida pela divisão $\mathrm{nSx}^{2} / \mathrm{Sp}^{2}$ se manteve maior que o F crítico $(5,99)$ tabelado ao nível de significância de $\alpha=0,05(5 \%)$ para todos os componentes (Tabela 2), apontando diferenças significativas entre os ambientes carbonáticos estudados.

A rejeição da hipótese nula $(\mathrm{F}>\mathrm{F}$ crítico) relaciona-se aos parâmetros $\mathrm{Ca}^{+2}, \mathrm{Mg}^{+2}, \mathrm{HCO}_{3}{ }^{-} \mathrm{e}$ $\mathrm{SO}_{4}^{-2}$ (Tabela 3).

Analisando o nível de significância adotado, o valor-p para os quatro parâmetros apresenta-se inferior ao $\alpha$, comprovando a rejeição da hipótese nula (Ho) e apontando diferenciação hidroquímica entre os três ambientes.

\section{Teste de Tukey}

Para verificar quais componentes químicos são responsáveis pelas diferenciações apresentadas, foram analisados os valores-p que apresentam índices inferiores ao nível de significância $(\alpha=0,05)$ e que rejeitam a hipótese nula $\left(\mathrm{H}_{\mathrm{o}}\right)$ (Tabela 4$)$.

$\mathrm{O} \mathrm{Mg}^{+2} \mathrm{e} \mathrm{HCO}_{3}{ }^{-}$apresentam valores-p inferior ao nível de significância em todos os ambientes, o que indica distinção entre as medianas das Formações (Tabela 4). Já no caso do $\mathrm{Ca}^{+2}$, existem diferenças entre as medianas das Fm. Sete Lagoas / Lagoa do Jacaré dos demais ambientes.

Tabela 3 - Análise de variância Kruskal-Wallis para os parâmetros $\mathrm{Ca}^{+2}, \mathrm{Mg}^{+2}, \mathrm{HCO}_{3}{ }^{-} \mathrm{e} \mathrm{SO}_{4}$.

\begin{tabular}{c|c|c|c}
\hline $\mathbf{C a}^{+\mathbf{2}}$ & Valor & $\mathbf{M g}^{\mathbf{+ 2}}$ & Valor \\
\hline $\mathbf{F}$ & 55,5 & $\mathrm{~F}$ & 174,5 \\
\hline Graus de Liberdade & 2 & Graus de Liberdade & 2 \\
\hline P-valor $^{-\mathbf{H C O}^{-}}$ & $9.10-^{-10}$ & P-valor & $5,5.10^{-8}$ \\
\hline $\mathbf{F}$ & Valor & $\mathbf{S O}^{-2}$ & Valor \\
\hline Graus de Liberdade & 20,9 & $\mathrm{~F}$ & 20 \\
\hline P-valor & 2 & Graus de Liberdade & 2 \\
\hline
\end{tabular}

A saturação de íons em solução é afetada pela solubilidade dos minerais, que, por sua vez, é influenciada pela composição das litofácies. A presença de sílica (feldspato alcalino, willemita e quartzo), por exemplo, contribui para a rocha se tornar menos susceptível à interação água/rocha, afetando a liberação de íons como $\mathrm{Ca}^{+2}, \mathrm{Mg}^{+2} \mathrm{e}$ $\mathrm{HCO}_{3}{ }^{-}$na água (Cunha, 2018). Nos ambientes estudados existem variações de concentração de sílica, apresentando na Fm. Olhos d'água 
intervalos entre 17,8 e $34,9 \mathrm{mg} / \mathrm{l}$, na Fm. Sete Lagoas/Lagoa do Jacaré intervalos entre 2,4 e $126 \mathrm{mg} / \mathrm{l}$ e Fm. Salitre intervalos entre 3,4 e 84 $\mathrm{mg} / \mathrm{l}$, corroborando para dissoluções distintas dos componentes e para a diferenciação constatada.

Para o $\mathrm{SO}_{4}{ }^{-2}$, os resultados apontam que existe diferença entre as medianas da Fm. Sete Lagoas / Lagoa do Jacaré das medianas encontradas na Fm. Salitre (Tabela 4). Nos dois ambientes, o
$\mathrm{SO}_{4}{ }^{-2}$ origina-se da oxidação de sulfetos piritosos e calcopiritosos, espalhados entre os carbonatos da Fm. Sete Lagoas / Lagoa do Jacaré, principalmente na Fm. Lagoa do Jacaré 2, e entre os carbonatos da Fm. Salitre principalmente na Unidade B1 (Misi, 1979; Misi et al., 2000; Nossa, 2011). As diferentes localizações do $\mathrm{SO}_{4}{ }^{-}$ 2 podem refletir na variação de seu teor nas águas analisadas.

Tabela 4 - Teste de Tukey para $\mathrm{Ca}^{+2}, \mathrm{Mg}^{+2}, \mathrm{HCO}_{3}{ }^{-}$e $\mathrm{SO}_{4}{ }^{-2}$.

\begin{tabular}{|c|c|c|c|}
\hline $\mathrm{Ca}^{+2}$ & P-valor & $\mathbf{M g}^{+2}$ & $\begin{array}{c}\text { P- } \\
\text { valor }\end{array}$ \\
\hline Fm Salitre/ Sete Lagoas e Lagoa do Jacaré & $\underset{10}{2,5.10^{-}}$ & Fm Salitre/ Sete Lagoas e Lagoa do Jacaré & $8.10^{-6}$ \\
\hline Fm Olhos d'água e Jacoca/Sete Lagoas e Lagoa do Jacaré & $2,3 \cdot 10^{-3}$ & Fm Olhos d'água e Jacoca/Sete Lagoas e Lagoa do Jacaré & $5,6.10^{-9}$ \\
\hline Fm Olhos d'água e Jacoca/Fm Salitre & 0,6 & Fm Olhos d'água e Jacoca/Fm Salitre & $5,6.10^{-3}$ \\
\hline $\mathrm{HCO}_{3}^{-}$ & P-valor & $\mathrm{SO}_{4}^{-2}$ & $\begin{array}{c}\text { P- } \\
\text { valor }\end{array}$ \\
\hline Fm Salitre/Fm Sete Lagoas e Lagoa do Jacaré & $3,2.10^{-2}$ & Fm Salitre/Fm Sete Lagoas e Lagoa do Jacaré & $4,9 \cdot 10^{-3}$ \\
\hline $\begin{array}{c}\text { Fm Olhos d'água e Jacoca / Fm Sete Lagoas e Lagoa do } \\
\text { Jacaré }\end{array}$ & $1,1.10^{-6}$ & $\begin{array}{c}\text { Fm Olhos d'água e Jacoca / Fm Sete Lagoas e Lagoa do } \\
\text { Jacaré }\end{array}$ & 0,2 \\
\hline Fm Olhos d'água e Jacoca/Fm Salitre & $5,7.10^{-5}$ & Fm Olhos d'água e Jacoca/Fm Salitre & 0,7 \\
\hline
\end{tabular}

\section{CONCLUSÃO}

Os resultados indicaram que as Fm. Olhos d'água/Jacoca apresentaram, em geral, maiores concentrações dos componentes químicos, dureza, condutividade elétrica (salinidade) e assinatura geoquímica apontando tendências cloretadas e sódicas na solução. A regressão múltipla indicou que condutividade elétrica apresentaram forte correlação com o $\mathrm{Na}^{+}$e o $\mathrm{Cl}^{-}$, sugerindo influência significativa da pluviométrica na salinidade dessas águas subterrâneas.

As Fm Sete Lagoas/Lagoa do Jacaré apresentaram menor concentrações dos componentes químicos, dureza e, em geral, menor salinidade, com assinatura geoquímica indicando tendências bicarbonatadas e cálcicas. A regressão múltipla indicou que o $\mathrm{Na}^{+}, \mathrm{Cl}^{-}, \mathrm{SO}_{4}{ }^{-}$ ${ }^{2}{\mathrm{e} \mathrm{HCO}_{3}}^{-}$possuem forte correlação com a condutividade elétrica, sugerindo contribuição de componentes litológicos e pluviométricos no teor de salinidade.

A Fm Salitre apresentou concentrações intermediárias dos componentes químicos e a assinatura geoquímica indicou tendências bicarbonatadas e cálcicas na solução, com influência magnesiana. A regressão múltipla indicou forte correlação entre a $\mathrm{CE}$ e o $\mathrm{Mg}^{2}$, sugerindo maior influência de componentes litológica para a salinidade das águas subterrâneas desse ambiente.

Os resultados estatísticos indicaram que os três ambientes carbonáticos são diferentes entre si. Através do teste de Tukey, constatou-se que as diferenças consistem na presença do $\mathrm{Mg}^{+2} \mathrm{e}$ $\mathrm{HCO}_{3}{ }^{-2}$. Com relação ao $\mathrm{Ca}^{+2}$, existe diferenças entre as Fm Sete Lagoas/Lagoa do Jacaré e as demais Formações. Estes resultados foram associados as diferentes distribuições mineralógicas nas litofácies, principalmente sílica, que afetam na dissolução dos componentes.

Por fim, no caso do $\mathrm{SO}_{4}{ }^{-2}$, ocorre diferenças entre as Fm Sete Lagoas/Lagoa do Jacaré e a Fm Salitre, possivelmente associado às variações de concentração de sulfetos piritoso e calcopiritosos nas áreas.

\section{REFERÊNCIAS}

APELLO, J.A.C. \& POSTMA, D. Geochemistry, Groundwater and Pollution. $2^{\mathrm{a}}$ ed. A.A. Balkema Publishers, p. 198 - 20, 2005. APHA. American public health association. Métodos Padrão para o Exame de Águas Residuais de Extração de Água. 1995, 19a edição. Washington, D. C. Disp. em: < http://www.sciepub.com/reference/217079>. Acesso em: 19 jan. 2019.
BARBOSA, J.S.F. \& DOMINGUEZ, J.M.L. Geologia da Bahia: texto explicativo para o mapa geológico ao milionésimo. Salvador, Secretaria da Indústria, Comércio e Mineração. Superintendência de Geologia e Recursos Minerais, 382p., 1996.

BAUER, L. Estimação do coeficiente de correlação de Spearman ponderado. Rio Grande do Sul, 2007. 10-12 p. 
Dissertação (Mestrado em Geologia). Instituto de Geociências. Universidade Federal do Rio Grande do Sul.

CARRERA-VILLACRÉS， D.; HIDALGO-HIDALGO， A.; GUEVARA, P.; VIVERO-BALAREZO, M.; DELGADO, V. Hydrogeochemistry Characterization of Hot Springs Located in The Andes of Ecuador. IOP Conference Series: Earth and Environmental Science, v. 4, n. 2, p. 3-8, 2016.

CARVALHO, V.J. Hidrogeoquímica e isótopos estáveis das águas subterrâneas do Aquífero Bambuí (Bahia, Brasil). Salvador, 2018. 20-30p. Dissertação (Mestrado em Geologia). Instituto de Geociências, Universidade Federal da Bahia.

COOPER, J.M.; COOPER, A.; CLAUSEN, B.; NICK, K. Regional bedrock geochemistry associated with podoconiosis evaluated by multivariate analysis. Environmental Geochemistry and Health, v. 41, n. 1, p. 04-12, 2018.

CUNHA, P. M. Interação água/rocha mineralizada em Fe, Pb, Zn e Ba, na porção Sul da Bacia Sedimentar de Irecê, Bahia, Brasil: Uma abordagem experimental. Salvador, 2018. 8487p. Tese (Doutorado em Geologia) - Instituto de Geociências, Universidade de Federal da Bahia.

CUSTODIO, E. \& LLAMAS, M.R. Hidrologia subterrânea Editora Omega, S.A, Barcelona, 2350p., 2001.

DINIZ, L. G. O Flúor nas águas subterrâneas do Estado de Minas Gerais. Belo Horizonte, 2006. 163 p. Dissertações (Mestrado em Geologia), Instituto de Geociências Universidade Federal de Minas Gerais.

FIELD, A. Descobrindo a estatística usando o SPSS. $2^{\text {a }}$ edição. Artmed. Porto Alegre, 298- 302 p., 2009.

GALVÃO, P.; HIRATA, R.; HALIHAN, T.; TERADA, R. Recharge sources and hydrochemical evolution of an urban karst aquifer, Sete Lagoas, MG, Brasil. Environmental Earth Sciences, v. 76, n. 4, p. 11-16, 2017.

GONÇALVES, P. V. M. Flúor nas águas subterrâneas do aquífero Bambuí no sudoeste da Bahia (Brasil). Brazilian Journal of aquatic Science and technology (BJAST), v. 22, n. 1, p. 10-15, 2018.

GONÇALVES, P. V. M. Flúor nas águas subterrâneas do aquífero Bambuí no sudoeste da Bahia (Brasil). Salvador, 2014. 26-27p. Tese (Doutorado em Geologia) - Instituto de Geociências, Universidade Federal da Bahia.

GONÇALVES, S.J.M. Glossário de Hidrologia. Gráfica Santa Helena. Salvador, 211 p., 2002.

GUERRA, A.M. Processos de carstificação e hidrogeologia do grupo Bambuí na região de Irecê - Bahia. São Paulo, 1986. 100-110 p. Tese (Doutorado em Geologia) - Instituto de Geociências, Universidade de São Paulo.

HUMPHREY, F.L. \& ALLARD, G.O. Geologia do domo de Itabaiana (Sergipe) e sua relação com a geologia do Geossinclinal de Propriá, um elemento tectônico recém reconhecido do escudo brasileiro. Rio de Janeiro: Petrobras/CENPES, 1969. $104 \mathrm{p}$

KIM, Y.; KIM, J. Y.; KIM, K. Geochemical Characteristics of Fluoride in Groundwater of Gimcheon, Korea: litogenic and agricultural origuns. Environ Moint Assess, v. 63, n.5, p. 1139-1148, 2011

LIMA, O.N.B. Grupo Bambuí: Estratigrafia regional no Alto Rio São Francisco e geologia dos depósitos fosfáticos da Serra da Saudade - MG. Minas Gerais, 2005. 142p. Dissertação (Mestrado em Geologia), Instituto de Geociências, Universidade Federal de Minas Gerais.

LIMA, S.A.A. Fácies, ambiente deposicional e aspectos diagenéticos das rochas carbonáticas da Formação Sete Lagoas na região norte de Minas Gerais, Bacia do São Francisco. Minas Gerais, 1997. 121 p. Dissertação (Mestrado em Geologia), Instituto de Geociências, Universidade Federal de Ouro Preto.

MESTRINHO, P.S.S. Geoquímica das águas subterrâneas. Cap. 5.2. Hidrogeologia conceitos e aplicações. CPRM, p. 366-378, 2008.

MISI A.; KAUFMAN, J.A.; VEIZER, J.; POWIS, K.; AZMY, K.; BOGGIANI, C.; GAUCHER, C.; TEIXEIRA, G.B.J.;
SANCHES, L.A., IYER, S.S.S. Chemostratigraphic Correlation of Neoproterozoic successions in South America. Chemical Geology, v. 237, p. 161-185, 2007.

MISI, A. \& SILVA, M.G. Chapada Diamantina Oriental - Bahia: Geologia e Depósitos. SGM, Série de Roteiros Geológicos, Salvador, 194 p, 1996.

MISI, A. \& SOUTO, P. Controle estratigráfico das mineralizações de chumbo, zinco, fluor e bário no Grupo Bambuí, parte leste da Chapada de Irecê (Bahia). Revista Brasileira de Geociências, v. 5, n. 1, p. 30-45, 1975.

MISI, A. O Grupo Bambuí no Estado da Bahia. In: INDA, H.A.V. Geologia e recursos minerais do Estado da Bahia: Textos básicos. Salvador: CPRM, v.1, p. 119-154, 1979.

MISI, A.; IYER, S.S.; COELHO, S.E.C.; TASSINARI, G.C.C.; FRANCA-ROCHA, S.J.W.; GOMES, R,S.A.; CUNHA, A.I.; TOULKERIDIS, T.; SANCHES, L.A. A metalogenic evolution model for the lead-zinc deposits of the Meso and Neoproterozoic sedimentary basins of the São Francisco Cráton, Bahia and Minas Gerais, Brazil. Revista Brasileira de Geociências, v. 30, n. 2, p. 302-305, 2000.

NASCIMENTO, M.A.S.; LEAL, B.R.L.; PURIFICAÇÃO, C.G.C. A utilização da análise de variância (ANOVA) na distinção de aquíferos sedimentares na região do Recôncavo Norte, Estado da Bahia. Revista Águas subterrâneas, v. 30, n. 3, p. 411-426, 2016.

NEGRÃO, I.F. Hidrogeologia do Estado da Bahia: Qualidade, potencialidade, disponibilidade, vulnerabilidade e grau de poluição. Espanha, 2007. 8-34 p. Tese (Doutorado em Geologia), Instituto Universitário de Xeoloxía Isidro Parga Pondal - Universidade de Coruña.

NOBRE-LOPES, J. Faciologia e Gênese dos Carbonatos do Grupo Bambuí na região de Arcos, Estado de Minas Gerais. São Paulo, 1995. 166 p. Dissertação (Mestrado em Geologia) Instituto de Geociências, Universidade de São Paulo.

NOSSA, B. C.T.; Avaliação da vulnerabilidade do aquífero cárstico Salitre - Bahia, através de análises hidroquímicas, isotópicas e aplicação da metodologia COP. Salvador, 211. 97-98p. Tese (Doutorado em Geologia) - Instituto de Geociências. Universidade Federal da Bahia.

OLIVEIRA, B.I; NEGRÃO, I.F.; SILVA, S.L.G.A. Mapeamento dos aquíferos do Estado da Bahia utilizando o índice de qualidade natural das águas subterrâneas - IQNAS. Revista de águas subterrâneas, v. 21, n. 1, p. 126, 2007.

RAMAGE, L. Hidrogeoquímica do sistema aquífero granular cenozoico do município de Porto Alegre, RS. Porto Alegre, 2005. 111p. Dissertação (Mestrado em Geologia), Instituto de Geociências - Universidade Federal do Rio Grande do Sul.

SALES, Q.L. Hidrogeologia e risco geológico em rochas carbonáticas Proterozoicas: porção central da Chapada Diamantina, Bahia, Brasil. Salvador, 2017. 43-55p. Tese (Mestrado em Geologia), Instituto de Geociências Universidade Federal da Bahia.

SAMPAIO, A.R.; ROCHA, A.J.D; SANTOS, R.A.; GUIMARAES, J.T. Programa Levantamentos Geológicos Básicos do Brasil - PLGB. Jacobina - Folha SC 24-y-C, Estado da Bahia, Escala 1:250.000. Brasília: CPRM/DIEDIG/DEPAT, 31p., 2001

SANTOS, C.P. Análise dos processos de salinização das águas subterrâneas da bacia do rio Salitre por meio de traçadores ambientais. Salvador, 2008. 125 p. Tese (Doutorado em Geologia), Instituto de Geociências - Universidade Federal da Bahia.

SANTOS, F.E. Caracterização Hidrogeológica e Hidroquímica da Bacia do Rio Verde Grande em Minas Gerais. Minas Gerais, 2013. 15-20 p. Dissertação (Mestrado em Geologia), Instituto de Geociências - Universidade Federal de Minas Gerais.

SANTOS, R. A. Hidrogeoquímica dos domínios cársticos de Irecê, Bahia - Brasil. Salvador, 2017. 29-40 p. Tese (Doutorado em Geologia), Instituto de Geociências Universidade Federal da Bahia, Bahia. 
SIAL, A.N.; GAUCHER, C.; SILVA FILHO, M.A.; FERREIRA, V.P.; PIMENTEL, M.M.; LACERDA L.D.; SILVA FILHO, E.V.; WILKER CEZARIO, W. C-, Sr-isotope and $\mathrm{Hg}$ chemostratigraphy of Neoproterozoic cap carbonates of the Sergipano Belt, Northeastern Brazil. Precambrian Research, v. 182, p. 351-372, 2010.

SILVA F.M.A.; BOMFIM, L.F.C.; SANTOS, R.A. Geologia da geossinclinal sergipana e do seu embasamento: Projeto Baixo São Francisco/Vaza Barris. Brasília: DNPM, 131 p. I, 1979.

SILVA, B.A. Hidrogeologia de meios cársticos. Capítulo 3.3. Hidrogeologia conceitos e aplicações. CPRM, 153-155p., 2008.

UHLEIN, A.; CAXITO, A.F.; SANGLARD, D.C.J.; UHLEIN, J.G.; SUCKAU, L.G. Estratigrafia e tectônica das faixas neoproterozoicas da porção norte do Cráton do São Francisco. Geonomos, v. 19, n. 2, p. 25, 2011.
VILLANUEVA, T.C.B.; LEAL, L.R.B.; ZUCCHI, M.R.; AZEVEDO. A.E.G.; VILLANUEVA P.R. Caracterização hidroquímica e hidrogeológica do aquífero cárstico salitre na região de Irecê, Bahia. Revista Brasileira de Recursos Hídricos, v.19, n.4, p. 87- 90, 2014. 